\section{ORIGINAL RESEARCH}

\section{M.M. Schneider \\ J.I. Berman \\ F.M. Baumer \\ H.C. Glass \\ S. Jeng \\ R.J. Jeremy \\ M. Esch \\ V. Biran \\ A.J. Barkovich \\ C. Studholme \\ D. $\mathrm{Xu}$ \\ O.A. Glenn}

\title{
Normative Apparent Diffusion Coefficient Values in the Developing Fetal Brain
}

BACKGROUND AND PURPOSE: Previous studies of diffusion-weighted imaging (DWI) in fetuses are limited. Because of the need for normative data for comparison with young fetuses and preterm neonates with suspected brain abnormalities, we studied apparent diffusion coefficient (ADC) values in a population of singleton, nonsedated, healthy fetuses.

MATERIALS AND METHODS: DWI was performed in 28 singleton nonsedated fetuses with normal or questionably abnormal results on sonography and normal fetal MR imaging results; 10 fetuses also had a second fetal MR imaging, which included DWI. ADC values in the periatrial white matter (WM), frontal WM, thalamus, basal ganglia, cerebellum, and pons were plotted against gestational age and analyzed with linear regression. We compared mean ADC in different regions using the Tukey Honestly Significant Difference test. We also compared rates of decline in ADC with increasing gestational age across different areas by using the $t$ test with multiple comparisons correction. Neurodevelopmental outcome was assessed.

RESULTS: Median gestational age was 24.28 weeks (range, 21-33.43 weeks). Results of all fetal MR imaging examinations were normal, including 1 fetus with a normal variant of a cavum velum interpositum. $A D C$ values were highest in the frontal and periatrial WM and lowest in the thalamus and pons. ADC declined with increasing gestational age in periatrial WM $(P=.0003)$, thalamus $(P<.0001)$, basal ganglia $(P=.0035)$, cerebellum $(P<.0001)$, and pons $(P=.024)$. Frontal WM ADC did not significantly change with gestational age. ADC declined fastest in the cerebellum, followed by the thalamus.

CONCLUSIONS: Regional differences in nonsedated fetal ADC values and their evolution with gestational age likely reflect differences in brain maturation and are similar to published data in premature neonates.

$\mathbf{S}^{\mathrm{s}}$ udies on diffusion-weighted imaging (DWI) in infants nd children have clearly demonstrated a progressive decline in water diffusivity in the brain, which parallels known histologic and functional data about brain development. Increasing interest in fetal therapy combined with the increasing incidence of premature births ${ }^{1,2}$ has resulted in an increasing need to understand normal brain maturation before term, yet, to our knowledge, there are few studies of diffusivity values in normal fetuses or in preterm infants before term. ${ }^{3-10}$ Preterm neonates are much more commonly studied at term equivalent age, and their diffusion metrics-apparent diffusion coefficient (ADC), fractional anisotropy, and eigenvalues-are compared with those of term neonates. As a result, few normal values have been generated for comparison with brain diffusion metrics of fetuses or preterm neonates with suspected brain abnormalities.

Limited studies of DWI in fetuses suggest that cerebral maturation is reflected in an evolution of ADC values that

Received February 20, 2009; accepted after revision April 5

From the Departments of Radiology and Biomedical Imaging (M.M.S., J.I.B., F.M.B., M.E., A.J.B., C.S., D.X., O.A.G.), and Neurology (H.C.G., S.J., V.B., A.J.B.); and Pediatric Clinical Research Center (R.J.J.), Department of Pediatrics, University of California, San Francisco, Calif.

This study was supported by the National Institutes of Health (NIH) grant K23 NS52506-03 and NIH/NCRR UCSF-CTSI grant UL1 RR024131. Its contents are solely the responsibility of the authors and do not necessarily represent the official views of the NIH.

Please address correspondence to Orit A. Glenn, MD, UCSF Department of Radiology and Biomedical Imaging, Neuroradiology Section, 505 Parnassus Ave, Box \#0628, San Francisco, CA 94143-0628; e-mail: orit.glenn@radiology.ucsf.edu

Indicates open access to non-subscribers at www.ajnr.org

DOI 10.3174/ajnr.A1661 begins in utero. ${ }^{3-6}$ These studies offer much promise to the study of premature neonates as well as to developmental abnormalities that may occur in utero. All such studies to date, however, are primarily on older fetuses, with very few fetuses imaged before 24 gestational weeks. Moreover, these studies are also limited in that they include fetuses with conditions known to affect brain development, such as twinning complications and congenital heart disease, ${ }^{11,12}$ fetuses with extracerebral malformations, and sedated fetuses, and have limited or no neurodevelopmental follow-up. To overcome these issues and to provide normative data for diffusivity of the developing preterm brain, we chose to study normative fetal ADC values in a population of singleton, nonsedated, healthy fetuses with normal neurodevelopmental outcome.

\section{Materials and Methods}

We identified all studies in which fetal DWI was performed as part of the fetal MR imaging from January 1, 2004, to November 1, 2008, in fetuses with normal prenatal ultrasonographic results or questionable sonographic abnormalities, with subsequent normal fetal MR imaging results. Fetal DWI was performed during maternal breath-hold on a 1.5T scanner (GE Healthcare, Milwaukee, Wis) with a single-shot spin-echo echo-planar DWI sequence acquired in the axial plane by use of a b-value of 0 and $600 \mathrm{~s} / \mathrm{mm}^{2}$ in 3 orthogonal directions. The following parameters were used: TR, $4500 \mathrm{~ms}$; TE, $\mathrm{min}$; FOV, $32 \mathrm{~cm}$; matrix, $128 \times 128$; section thickness, $5 \mathrm{~mm}$; skip, $2 \mathrm{~mm}$; and bandwidth, $167 \mathrm{kHz}$. As part of the fetal MR imaging, all women also had 3-mm axial, sagittal, and coronal single-shot fast spin-echo T2weighted images acquired with use of real-time imaging for the fetus ${ }^{13}$ and axial fast multiplanar spoiled gradient-recalled acquisition in the steady state T1-weighted images. In cases in which a second fetal MR 

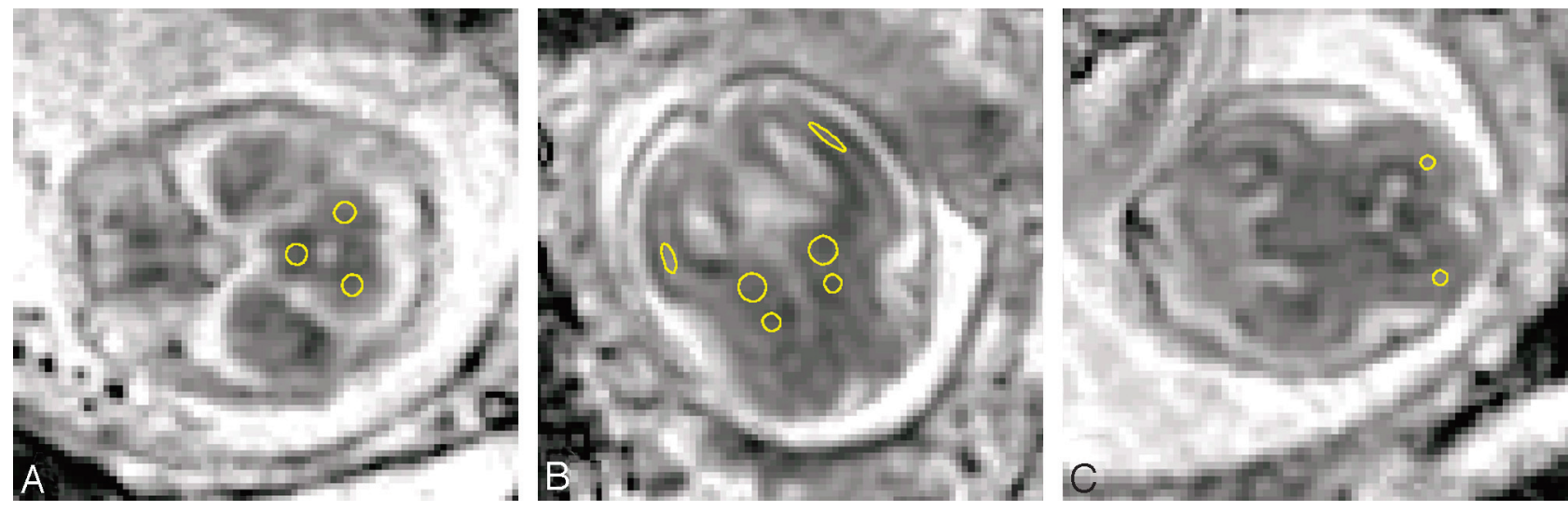

Fig 1. $A D C$ map in fetuses aged 22.43 to 23 weeks showing ROls in the $(A)$ cerebellum and pons; $(B)$ periatrial WM, thalamus, and basal ganglia; and $(C)$ frontal WM.

imaging examination was performed as part of the evaluation, the MR imaging and DWI techniques were the same as for the first fetal MR imaging. All fetal MR images were performed without any maternal or fetal sedation. Our institutional review board approved this study.

Fetal DWI was transferred off-line, and each diffusion image was reviewed by a pediatric neuroradiologist for any evidence of motion in any direction or other artifacts such as from bowel gas in the maternal rectum. When a raw diffusion image was affected by motion or other artifacts, any data from that section were excluded from the study. When fetuses had more than 1 diffusion series per scan, every available diffusion series was evaluated in this manner. We then calculated ADC maps by using in-house software and also reviewed ADC maps for image quality such as distinction of the germinal matrix and cortex from adjacent developing white matter (WM) and CSF. Because diffusion gradients were only applied in 3 directions, we could only measure $\mathrm{ADC}$ values and could not measure other diffusion parameters such as anisotropy.

Regions of interest (ROIs) were drawn on ADC maps in the periatrial WM, frontal WM, thalamus, basal ganglia, cerebellum, and pons (Fig 1). Whenever possible, ROIs were placed bilaterally. ROIs were always reviewed by a pediatric neuroradiologist. For the thalamus, basal ganglia, and pons, a circular ROI was used, which included as much of the anatomic area of interest as possible. ROI areas ranged from 17 to $41 \mathrm{~mm}^{2}$ for the thalamus, 8 to $45 \mathrm{~mm}^{2}$ for the basal ganglia, and 7 to $24 \mathrm{~mm}^{2}$ for the pons. The cerebellar ROI was placed in the center of the cerebellum to minimize inclusion of the cortex, with ROI areas ranging from 9 to $60 \mathrm{~mm}^{2}$. Both periatrial WM ROI and frontal WM ROI extended across the cerebral mantle, sparing the germinal zone and the cortex; therefore, they included the intermediate zone and the subplate, both of which will ultimately contribute to the cerebral WM. The periatrial WM ROI was ovoid and was placed midway between the anterior and posterior margin of the ventricular atrium, and its area was proportional to the width of the brain at the same section. The width of the brain was multiplied by a constant factor of 0.025 , and the resulting product was used as the area of the periatrial WM ROI, with areas ranging from 10 to $20 \mathrm{~mm}^{2}$. The frontal WM ROI was circular and was drawn anterior to the frontal horns on the most inferior section through the frontal horns. Frontal WM ROIs ranged in size from 4 to $64 \mathrm{~mm}^{2}$.

We calculated ADC values for each anatomic ROI using in-house software. When ROIs were available for more than 1 diffusion series acquisition for a fetus, $\mathrm{ADC}$ values were averaged for all the diffusion series to yield 1 value for each anatomic location. Because ADC values were not significantly different between the right and left hemispheres with use of the Wilcoxon signed-rank test, ADC values from both sides were averaged for each anatomic location when available.

For each of the anatomic locations, ADC values were plotted against gestational age by last menstrual period and analyzed with linear regression $(P<.05)$. The linear regression model was not significantly affected by a variable classifying the fetuses scanned at 2 time points. Thus, multiple scans of the same fetus were included as separate data points in our analyses. We compared mean ADC values in the different anatomic regions using the Tukey Honestly Significant Difference test $(P<.05)$. We then compared rates of decline in ADC values with increasing gestational age across different areas with a $t$ test by using the Bonferroni correction for multiple comparisons, with a corrected $P$ value of .0125 .

Neurodevelopmental outcome was assessed by either a phone interview and questionnaire with the parents or by a structured neurologic examination with the neuromotor score ${ }^{14}$ by a child neurologist and the Bayley Scales of Infant and Toddler Development, 3rd edition, ${ }^{15}$ administered by a research psychologist at 1 year postnatal age. A neuromotor score of 0 or 1 and Bayley-III Cognitive, Language, and Motor Scale scores of 80 or more were considered normal. For those evaluated by phone questionnaire, the child's development was considered normal if the child met developmental milestones with no developmental concerns by the pediatrician and parent. Only children with normal neurodevelopment were included in this study.

\section{Results}

A total of 45 fetuses had DWI performed as part of fetal brain MR imaging; in 17 cases, a second fetal MR imaging examination was also performed. Of these, 28 fetuses were included in our study, 10 of whom also had a second fetal MR imaging, for a total of 38 fetal MR imaging examinations contributing to the data in this report. Median gestational age by last menstrual period at the time of the MR imaging was 24.28 weeks (range, 21-33.43 weeks), with 16 fetuses scanned before 24 gestational weeks. A total of 13 women were scanned as healthy volunteers as part of an ongoing fetal MR imaging study, and 2 of those also had a previous abnormal pregnancy; all women had normal prenatal sonographic results before the MR imaging study, including 1 in whom a small enteric duplication cyst was incidentally detected by fetal MR imaging. A total of 8 women were scanned because of a previous pregnancy with brain abnormalities, and all had normal prenatal ultrasonographic results except for 1 patient, who had a single multicystic dysplastic kidney. There were 7 women who un- 
derwent scanning because of questionable sonographic abnormalities. These included 4 cases with possible inferior vermian hypogenesis on an early ultrasonographic study and then a normal-appearing vermis on subsequent ultrasounds performed before the fetal MR imaging, and 3 cases with a questionable midline cyst vs a prominent posterior third ventricle ( 1 of whom had normal results on ultrasound on a more detailed level II sonography examination before the MR imaging, and 1 of whom had a cavum velum interpositum on fetal MR imaging). All women had normal fetal MR imaging results, including 1 fetus with a normal variant of a cavum velum interpositum. Mean age at postnatal assessment was 15 months (range, 3-58.4 months) and included Bayley-III and neurologic evaluation in 8 cases, phone questionnaire in 18 cases, and ongoing pregnancies in 2 cases. All children met age-appropriate neurodevelopmental milestones. For the children who underwent formal neurologic evaluation at 1 year postnatal age, mean scores for the Bayley-III Cognitive, Language, and Motor Scales were 104, 89, and 96, respectively.

In each case, at least 2 DWI sequences were performed, with a mean number of 4.8 diffusion sequences per MR imaging examination. Of the total of 38 scans included in our study, a periatrial WM ROI was successfully drawn on 33 scans; a basal ganglia ROI, on 28 scans; a thalamus ROI, on 28 scans; a frontal WM ROI, on 26 scans; a cerebellum ROI, on 16 scans; and a pons ROI, on 17 scans.

The highest ADC values occurred in the frontal and periatrial WM, and they were significantly higher than all other areas (Fig 2). The cerebellum had significantly higher ADC values than the basal ganglia, thalamus, and pons. The lowest ADC values were observed in the thalamus and pons, and these ADC values were significantly lower than those in all other areas.

With increasing gestational age, a significant decline in ADC values was found in the periatrial WM $(P=.0003)$, thalamus $(P<.0001)$, basal ganglia $(P=.0035)$, cerebellum $(P<$ $.0001)$, and pons $(P=.024)$ (Fig 2$)$. We did not observe a significant change in ADC in the frontal WM with increasing gestational age. The cerebellum had the fastest rate of decline in $\mathrm{ADC}$ values with increasing gestational age, which was significantly faster than the periatrial WM $(P=.0001)$, frontal WM $(P=.000000)$, basal ganglia $(P=.000003)$, thalamus $(P=.012)$, and pons $(P=.009)$. ADC values declined more rapidly in the periatrial WM than in the frontal WM $(P=$ $.001)$, but were not significantly different from the thalamus, basal ganglia, or pons. The thalamus had the second fastest rate of decline of ADC values with increasing gestational age, and this was significantly faster than the basal ganglia $(P=$ $.002)$ and frontal WM $(P=.0000004)$.

\section{Discussion}

We observed regional differences in ADC values as well as different rates of decline in ADC with increasing gestational age in nonsedated singleton fetuses during the second half of the second trimester and the first half of the third trimester. In particular, ADC values in the supratentorial WM were higher than ADC values in the deep gray nuclei, cerebellum, and pons. Moreover, the most rapid decline in ADC values was observed in the cerebellum and thalamus, followed by the pons, basal ganglia, and periatrial WM, with no significant decline in the frontal WM.

Previous studies have shown a steady decline in ADC values in the supratentorial deep gray nuclei with increasing gestational age ${ }^{3,4,6}$ which is similar to what we observed. Changes in $\mathrm{ADC}$ values in the supratentorial WM, however, are more variable. ${ }^{3,4,6}$ In the first published study of DWI in fetuses aged 22 to 35 gestational weeks, ADC values in the periatrial and frontal WM did not significantly decline with increasing gestational age. ${ }^{3}$ However, this study was limited to 15 nonsedated fetuses, some of which were twin pregnancies complicated by twin-to-twin transfusion syndrome. Because sulcation is delayed in twins, ${ }^{16}$ it is likely that other markers of brain development are delayed as well, perhaps even more so in complicated monochorionic twin pregnancies. A subsequent study of 56 nonsedated fetuses did observe a decline in supratentorial WM ADC values ${ }^{6}$; however, they averaged the frontal and parietal WM values, and we have shown that ADC values and their change with increasing gestational age are different in the frontal WM and the parietal WM. Moreover, although they observed a decline in supratentorial WM ADC values with increasing gestational age, it is important to note that they included fetuses with cardiac abnormalities because cardiac abnormalities likely influence brain development. ${ }^{11}$

Fetal ADC values have also been reported in studies of sedated fetuses. ${ }^{4,5}$ Our observation of a decline in ADC values in the thalamus, pons, and cerebellum with increasing gestational age is similar to the largest study of fetal DWI in 78 sedated fetuses by Schneider et al. ${ }^{4}$ However, it is interesting to note that they observed an increase in ADC values in the periatrial and frontal WM and basal ganglia from 23 to 29 weeks' gestation, with a decrease in ADC values from 30 to 36 weeks' gestation. These differences in observation could be the result of the size and shape of ROIs because our ROIs were adjusted depending on the size of the fetal brain. In particular, our periatrial WM ROIs were ovoid, ranging in size from 10 to 20 $\mathrm{mm}^{2}$ depending on the diameter of the brain at that level, and did not include the cortex or germinal matrix. It is possible that the lower ADC values that they observed in younger fetuses could be the result of their fixed ROI size of $84 \mathrm{~mm}^{2}$ regardless of gestational age. In younger fetuses with thinner cerebral parenchyma, a large circular ROI could result in inclusion of the germinal matrix and/or cortex and, thus, lower ADC values. Moreover, these differences could also be from the greater number of young fetuses included in our study. In particular, we measured periatrial WM ADC values in $18 \mathrm{fe}$ tuses that were younger than 28 gestational weeks compared with 9 fetuses included in their study. Furthermore, only 2 of their fetuses were younger than 24 gestational weeks, which differs from our study, which includes 16 fetuses that were younger than 24 gestational weeks at the time of MR imaging.

It is worthy to note that because normal fetal values might conceivably be considered norms for preterm neonates, our findings are similar to what has been observed in premature infants scanned preterm. ${ }^{7,8,10}$ More specifically, our thalamic, basal ganglial, and posterior WM ADC values are similar to what has been reported in preterm neonates as young as 27 weeks with normal results on MR imaging and normal results at 1-year neurodevelopmental examination. ${ }^{10}$ Furthermore, $\mathrm{ADC}$ values also decline with increasing gestational age in pre- 


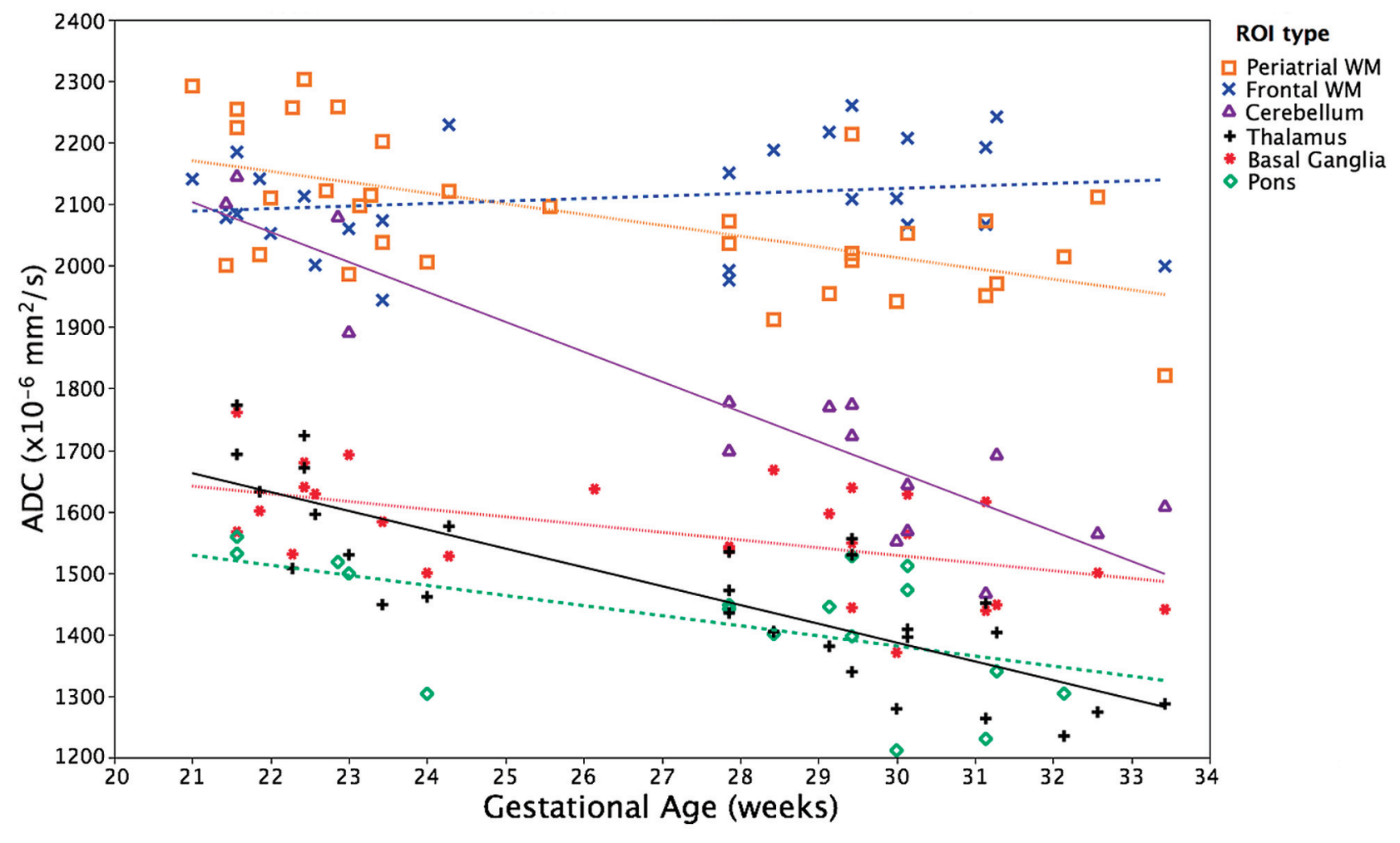

Fig 2. ADC vs gestational age for all ROls.

term neonates scanned at preterm. ${ }^{7,8,10}$ In premature neonates aged 27 to 38 weeks, the most rapid decline in ADC values was seen in the thalamus, followed by the basal ganglia, with the slowest decline in the parietal and frontal WM, which is very similar to what we observed. ${ }^{10}$

Our study differs from previously published fetal DWI studies in that many of our fetuses were less than 24 gestational weeks, which reflects the gestational period when most fetal MR imaging examinations are performed in the United States. Nearly all previous studies only included 2 to 6 fetuses younger than 24 gestational weeks, compared with 16 fetuses younger than 24 gestational weeks that were included in our study. Moreover, the mean age at follow-up was greater in our study compared with previous published studies in which neonatal or 6-month postnatal follow-up was performed; and nearly one third of our patients had formal neurologic evaluation and neurodevelopmental testing at 1 year postnatal age.

Our observed regional differences in ADC during gestation likely reflect differences in brain development because of many factors, including increasing cellularity, neuronal maturation, and myelination. Indeed, areas that are known to mature and myelinate earlier, such as the cerebellum, pons, and thalamus, had lower ADC values. The highest ADC values were observed in the supratentorial WM. One of the reasons for the relatively high ADC values is likely the result of inclusion of the subplate zone in our WM ROIs because the ROIs encompassed all of the cerebral mantle, sparing the germinal matrix zone and cortex. The subplate zone comprises a large amount of the developing cerebral mantle during most of the gestational ages examined in our study, reaching peak between 29 to 32 gestational weeks, ${ }^{17}$ and has been shown to have high ADC values. ${ }^{18}$ It is interesting to note that the subplate zone persists longer in association areas such as the frontal lobes (which also undergo late myelination and late sulcation), where we consistently observed the highest ADC values. It is important to note, however, that both the subplate zone and intermediate zone likely contributed to our WM ROIs, and the relative amount of contribution from each layer likely differed in different anatomic areas and with differing gestational age. Moreover, the composition of the subplate zone also changes in different anatomic areas of the brain with differing gestational age. ${ }^{19}$ Future studies with diffusion tensor imaging are needed to further explore the layers of the developing fetal brain.

Although fetal DWI is limited by fetal motion, our study demonstrates the feasibility of performing DWI in a large group of healthy nonsedated singleton fetuses, including those at younger gestational ages. This is important because fetal MR imaging in the United States is usually performed before 24 gestational weeks and is done without any maternal or fetal sedation. It is most important to stress that the similarity of our results with those of preterm infants scanned postnatally demonstrates that fetal DWI can be performed accurately in many nonsedated fetuses. This has implications both for the study of fetal brain development and for the study of brain injury in preterm infants. In most institutions, preterm infants are scanned at term equivalent age and are compared with normal-term neonates. Being able to scan preterm infants before term and compare their ADC values with normative fetal data may provide earlier, important information about their brain development. Moreover, these data could then be correlated with neurodevelopmental outcome, with the eventual goal of being able to predict outcome before these neonates reach term-equivalent age. 
Our study was somewhat limited by the effects of fetal motion and, less so, of maternal motion. Overall, 39\% of the scans that we initially assessed could not be used in our study. We found that only $42 \%$ to $53 \%$ of the scans were not affected by fetal motion or other artifacts at the level of the basal ganglia, thalamus, periatrial, and frontal WM and, thus, were included in our study. DWI at the level of the cerebellum and pons was more limited, usually because of fetal motion or exclusion of the cerebellum and pons in some of the sequences. Our study was also limited in that we were only able to perform DWI, and not diffusion tensor imaging, in our fetuses because of the longer image acquisition times for diffusion tensor imaging (and consequent greater fetal motion) and thus could not calculate other important diffusion measures such as anisotropy and individual eigenvalues. Recent studies have demonstrated some feasibility in performing fetal diffusion tensor imaging, ${ }^{20,21}$ though future technical improvements will hopefully allow faster data acquisition and more practical application of diffusion tensor imaging to young nonsedated fetuses.

\section{Conclusions}

In summary, we measured ADC values in normal, nonsedated singleton fetuses aged 21 to 33 gestational weeks. The values themselves, and the evolution of values with increasing gestational ages, differed for different areas of the brain and were similar to published data in premature neonates. This has implications for the study of premature neonates as well as developmental abnormalities that may occur in utero.

\section{Acknowledgment}

The authors would like to thank Ben Mow for scanning the patients and assistance with the protocol.

\section{References}

1. Cortes RA, Farmer DL. Recent advances in fetal surgery. Semin Perinatol 2004;28:199-211
2. Goldenberg RL, Culhane JF, Iams JD, et al. Epidemiology and causes of preterm birth. Lancet 2008;371:75-84

3. Righini A, Bianchini E, Parazzini C, et al. Apparent diffusion coefficient determination in normal fetal brain: a prenatal MR imaging study. AJNR Am J Neuroradiol 2003;24:799-804

4. Schneider JF, Confort-Gouny S, Le Fur Y, et al. Diffusion-weighted imaging in normal fetal brain maturation. Eur Radiol 2007;17:2422-29

5. Cannie M, De Keyzer F, Meersschaert J, et al. A diffusion-weighted template for gestational age-related apparent diffusion coefficient values in the developing fetal brain. Ultrasound Obstet Gynecol 2007;30:318-24

6. Manganaro L, Perrone A, Savelli S, et al. Evaluation of normal brain development by prenatal MR imaging. Radiol Med 2007;112:444-55

7. Huppi PS, Maier SE, Peled S, et al. Microstructural development of human newborn cerebral white matter assessed in vivo by diffusion tensor magnetic resonance imaging. Pediatr Res 1998;44:584-90

8. Neil JJ, Shiran SI, McKinstry RC, et al. Normal brain in human newborns: apparent diffusion coefficient and diffusion anisotrophy measured by using diffusion tensor MR imaging. Radiology 1998;209:57-66

9. Tanner SF, Ramenghi LA, Ridgway JP, et al. Quantitative comparison of intrabrain diffusion in adults and preterm and term neonates and infants $A J R A m J$ Roentgenol 2000;174:1643-50

10. Miller S, Vigneron DB, Henry RG, et al. Serial quantitative diffusion tensor MRI of the premature brain: development in newborns with and without injury. J Magn Reson Imaging 2002;16:621-32

11. Miller SP, McQuillen PS, Hamrick S, et al. Abnormal brain development in newborns with congenital heart disease. N Engl J Med 2007;357:1928-38

12. Quarello E, Molho M, Ville Y. Incidence, mechanisms, and pattern of fetal cerebral lesions in twin-to-twin transfusion syndrome. J Matern Fetal Neonatal Med 2007;20:589-97

13. Busse R, Carrillo A, Brittain J, et al. On-demand real-time imaging: interactive multislice acquisition applied to prostate and fetal imaging. International Society for Magnetic Resonance in Medicine, Honolulu, Hawaii; 2002

14. Hajnal B, Sahebkar-Moghaddam F, Barnwell A, et al. Early prediction of neurologic outcome after perinatal depression. Pediatr Neurol 1999;21:788-93

15. Bayley N. Bayley Scales of Infant and Toddler Development, Third Edition. San Antonio: Psychological Corporation of Harcourt Assessment; 2006

16. Chi J, Dooling E, Gilles F. Gyral development of the human brain. Ann Neurol 1977;1:86-93

17. Kostović I, Judas M, Rados M, et al. Laminar organization of the human fetal cerebrum revealed by histochemical markers and magnetic resonance imaging. Cereb Cortex 2002;12:536-44

18. Maas LC, Mukherejee P, Carballido-Gamio J, et al. Early laminar organization of the human cerebrum demonstrated with diffusion tensor imaging in extremely premature infants. Neuroimage 2004;22:1134-40

19. Kostović I, Jovanov-Milosević N. Subplate zone of the human brain: historical perspective and new concepts. Coll Antropol 2008;32 Suppl 1:3-8

20. Bui T, Daire JL, Chalard F, et al. Microstructural development of human brain assessed in utero by diffusion tensor imaging. Pediat Radiol 2006;36:1133-40

21. Kasprian G, Brugger P, Weber M, et al. In utero tractography of fetal white matter development. NeuroImage 2008;43:213-24 